of 2 patients with Omenn syndrome. J. Clin. Invest. 115:728-732. doi:10.1172/JCI200523087.

13. Marrella, V., et al. 2007. A hypomorphic R229Q Rag2 mouse mutant recapitulates human Omenn syndrome. J. Clin. Invest. 117:1260-1269. doi:10.1172/JCI30928.

14. Qiu, J.X., Kale, S.B., Yarnell Schultz, H., and Roth, D.B. 2001. Separation-of-function mutants reveal critical roles for RAG2 in both the cleavage and joining steps of V(D)J recombination. Mol. Cell. 7:77-87

15. Khiong, K., et al. 2007. Homeostatically proliferating $\mathrm{CD}^{+} \mathrm{T}$ cells are involved in the pathogenesis of an Omenn syndrome murine model. J. Clin. Invest. 117:1270-1281. doi:10.1172/JCI30513.

16. Lu, C.P., Sandoval, H., Brandt, V.L., Rice, P.A., and Roth, D.B. 2006. Amino acid residues in Rag1 crucial for DNA hairpin formation. Nat. Struct. Mol. Biol. 13:1010-1015

17. Min, B., Foucras, G., Meier-Schellersheim, M., and Paul, W.E. 2004. Spontaneous proliferation, a response of naive CD4 $\mathrm{T}$ cells determined by the diversity of the memory cell repertoire. Proc. Natl. Acad. Sci. U. S. A. 101:3874-3879.

18. Baccala, R., and Theofilopoulos, A.N. 2005. The new paradigm of T-cell homeostatic proliferationinduced autoimmunity. Trends Immunol. 26:5-8.

19. Curotto de Lafaille, M.A., Shen, S., Olivares-Villagomez, D., Camps-Ramirez, M., and Lafaille, J.J. 2005. Do regulatory T cells play a role in the control of homeostatic proliferation? Int. Rev. Immunol. 24:269-284.

20. Curotto de Lafaille, M.A., and Lafaille, J.J. 2004. The role of regulatory $\mathrm{T}$ cells in allergy. Springer Semin.
Immunopathol. 25:295-310.

21. Krupica, T., Jr., Fry, T.J., and Mackall, C.L. 2006. Autoimmunity during lymphopenia: a two-hit model. Clin. Immunol. 120:121-128.

22. Milner, J.D., Ward, J.M., Keane-Myers, A., and Paul, W.E. 2007. Lymphopenic mice reconstituted with limited repertoire $\mathrm{T}$ cells develop severe, multiorgan, Th2-associated inflammatory disease. Proc. Natl. Acad. Sci. U. S. A. 104:576-581.

23. Jankovic, M., Casellas, R., Yannoutsos, N., Wardemann, H., and Nussenzweig, M.C. 2004. RAGs and regulation of autoantibodies. Annu. Rev. Immunol. 22:485-501.

24. Aalberse, R.C., and Platts-Mills, T.A. 2004. How do we avoid developing allergy: modifications of the $\mathrm{TH} 2$ response from a B-cell perspective. J. Allergy Clin. Immunol. 113:983-986.

\title{
Cutaneous mosaicism: right before our eyes
}

\author{
Jorge Frank ${ }^{1,2}$ and Rudolf Happle ${ }^{3}$
}

1Department of Dermatology and 2Maastricht University Centre for Molecular Dermatology, University Hospital, Maastricht, The Netherlands. ${ }^{3}$ Department of Dermatology, Philipp University of Marburg, Marburg, Germany.

\begin{abstract}
Autosomal recessive cutaneous disorders, including various types of epidermolysis bullosa (EB), usually manifest shortly after birth. The clinical course of these diseases is often characterized by severe complications, limited therapeutic options, and a poor prognosis. A study by Pasmooij et al. reported in this issue of the JCI unravels the molecular mechanisms by which germline mutations in non-Herlitz junctional EB can be corrected in vivo by multiple spontaneously occurring somatic mutational events, a phenomenon known as revertant mosaicism (see the related article beginning on page 1240). These insights open new avenues of thinking for the design of future gene therapy strategies for skin diseases.
\end{abstract}

"I see a bright future for genetics in dermatology and for dermatology in genetics..." - this was the vision of Victor A. McKusick, widely regarded as one of the founding fathers of medical genetics, some 35 years ago (1). Today, it is justified to say that he was absolutely right. During the past 20 years, the genetic basis of more than 350 monogenic skin diseases has been elucidated (2), and the number of disorders awaiting discovery of the underlying gene defect is steadily increasing. However, research in the field of inherited cutaneous disorders is by no means limited to finding novel genes and mutations. Because the skin is easily accessible and, for the trained observer, everything lies "right before your eyes," genetic skin diseases offer unique oppor-

Nonstandard abbreviations used: EB, epidermolysis bullosa; LAMB3, laminin $\beta 3$; LOH, loss of heterozygosity.

Conflict of interest: The authors have declared that no conflict of interest exists.

Citation for this article: J. Clin. Invest. 117:1216-1219 (2007). doi:10.1172/JCI32111. tunities to study complex clinical expression patterns and uncommon inheritance modes and the ability to explore molecular therapeutic regimens such as enzyme replacement strategies and gene therapy $(2,3)$. This notion holds particularly true for cutaneous disorders that clinically present with mosaicism.

\section{Mosaicism}

The term mosaicism refers to the occurrence in an individual of two or more cell populations that are karyotypically or genotypically different and yet are derived from a single zygote (4). Mosaicism can result from a mutation during development that is propagated in only a limited number of the adult cells. In general, any type of cell may be affected by such a mutational event, including gametes (egg and sperm cells), blood cells, and skin cells. The best-known example of mosaicism is mammalian females (normal karyotype 46,XX), which are functional mosaics because one of their X chromosomes is randomly inactivated during embryogenesis (5).
In autosomal recessive mendelian disorders, the underlying mutations often cause embryonic or early postnatal death. Notwithstanding, studying the effects of such mutations at later developmental stages may be possible in individuals carrying the mutation in a mosaic form because gain or loss of genetic functions might be limited to specific cells and tissues or to selected stages of development, as recently shown in various organisms, including, for example, Caenorbabditis elegans, Drosophila, mice, and zebrafish (6-9). Therefore, mosaic organisms are perfectly suited for the investigation of the molecular processes orchestrating very early developmental phases. Further, they may be of use to study a specific cell type or tissue in which a given gene is required to assure proper functioning of signaling pathways and metabolic processes. With respect to organ systems, mosaic organisms can serve to determine whether a particular gene is cell autonomous, i.e., whether the gene is exerting its action exclusively within the cell in which it is expressed or if it also affects neighboring cells that do not manifest a phenotype themselves when carrying a mutation in that gene (8).

\section{Mosaicism in monogenic skin disorders}

Mosaic skin diseases may show different patterns of clinical involvement such as lines of Blaschko, a checkerboard pattern, a phylloid pattern, a patchy pattern without midline separation, and a lateralization 

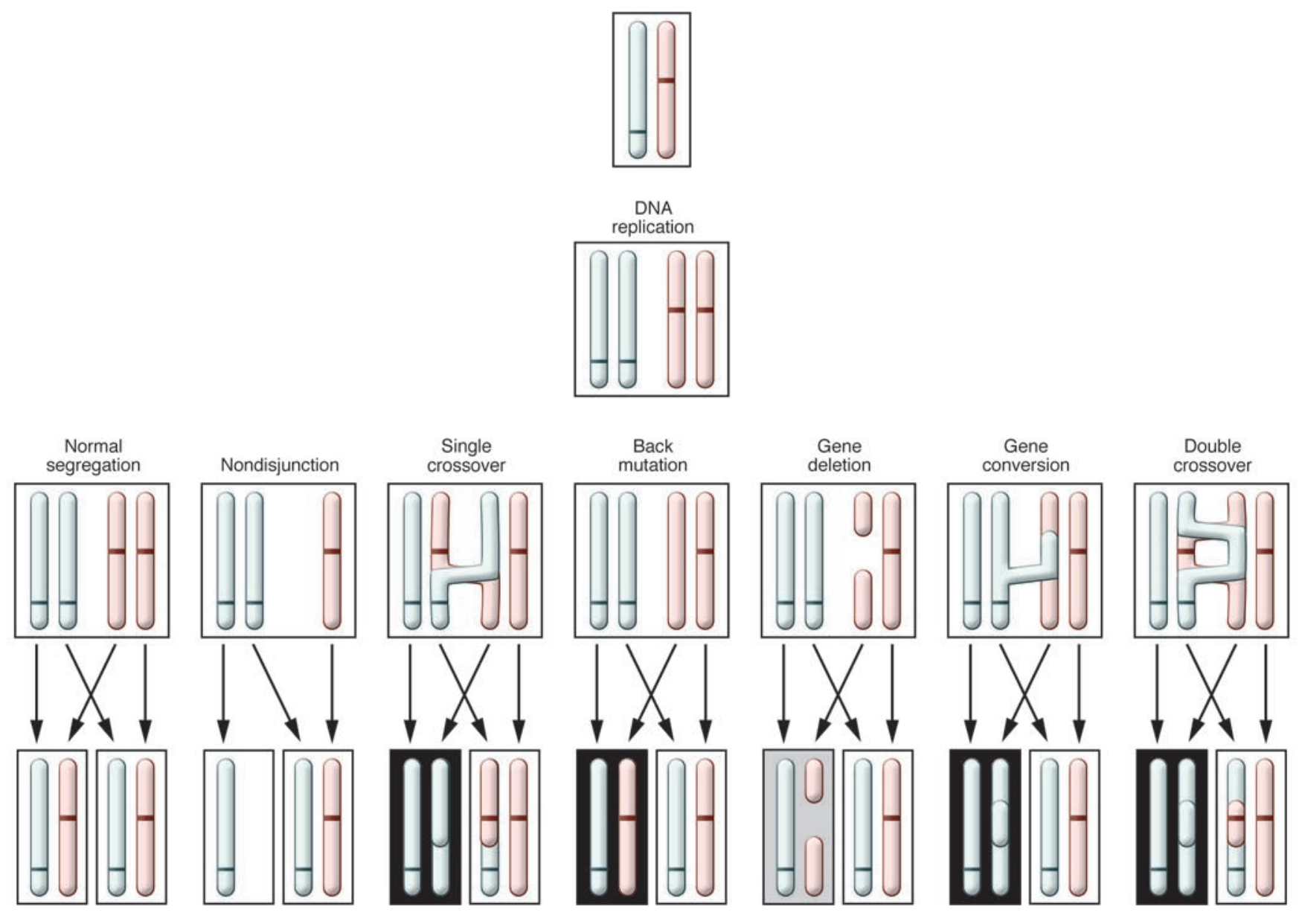

Figure 1

Schematic representation of postzygotic mechanisms that may give rise to revertant mosaicism in an individual compound heterozygous for an autosomal recessive phenotype. Two corresponding chromosomes carry either the paternal (blue) or maternal (pink) mutation. Various mutational events may result in cells showing loss of compound heterozygosity (bottom row). As a consequence, these cells will produce either a functional protein (black boxes), a semifunctional protein (gray box), or no protein at all (white boxes). Reproduced with permission from the American Journal of Medical Genetics (19).

pattern. Mosaicism has been demonstrated at the cellular level in more than 15 monogenic skin disorders to date (10). Recently, the specific molecular mechanisms governing three particular forms of cutaneous mosaicism have been unraveled and reported in the JCI.

In 2004, we showed at the cellular and molecular level that the type 2 segmental manifestation of Hailey-Hailey disease, being superimposed on the ordinary nonsegmental lesions of this autosomal dominant trait, results from postzygotic loss of heterozygosity (LOH) (11). These data provided the first molecular evidence supporting this genetic concept, which was postulated ten years ago (12). If $\mathrm{LOH}$ results from postzygotic crossing-over, this would give rise to two different daughter cells, one of them being homozygous for the underlying mutation. Conversely, the other cell would be homozygous for the wild-type allele, resulting in a band or patch of completely healthy tissue (13). This would reflect "natural gene therapy" in an autosomal dominant skin disease in a way analogous to that proposed by Pasmooij et al. in this issue of the JCI (14) for autosomal recessive cutaneous traits. After ex vivo expansion of cells from such healthy skin areas, cell sheets could be generated and used for autologous transplantation.

More recently, the molecular basis of linear epidermal nevi of the common, nonorganoid and nonepidermolytic type was also reported in the JCI (15). These benign keratinocytic skin lesions are present at birth but become more conspicuous during early childhood. Using a SNaPshot Multiplex assay, Hafner et al. detected activat- ing somatic mutations in the FGF receptor 3 (FGFR3) gene in about one-third of the nevi studied (15). These findings could provide a basis for the future development of noninvasive therapeutic strategies for skin tumors, such as, for example, siRNA specific to the mutant allele (16). A significant therapeutic benefit of RNA interference in vivo has recently been demonstrated for amyotrophic lateral sclerosis (17).

\section{Revertant mosaicism}

Over the last 20 years, the phenotypic reversion of a clinically severe autosomal recessively inherited disease by one or several correcting somatic mutations has been described in various human disorders. This particular category of human mosaicism was first described in Lesch-Nyhan syndrome in 1988 (18). Various molecular mechanisms 
governing this kind of naturally occurring phenotypic rescue have been proposed and demonstrated, including true reverse point mutations, deletions, nondisjunction, crossing-over events, and gene conversion (Figure 1) (19). Moreover, revertant mosaicism can be induced by transposable elements known as retrotransposons (20) as documented in Duchenne muscular dystrophy $(21,22)$.

In 1997, Jonkman et al. were the first to demonstrate at the molecular level the occurrence of revertant mosaicism in human skin in a patient suffering from generalized atrophic benign epidermolysis bullosa (EB), who showed some healthy patches of skin in which blistering could not be evoked (23). The authors proved that within these areas, phenotypic reversion was caused by mitotic gene conversion in one of the two mutated collagen type XVII $\alpha 1$ (COL17A1) alleles. Based on these data, they suggested that revertant mosaicism in autosomal recessive skin diseases is as an example of natural gene therapy.

\section{Revertant mosaicism in EB: guiding the path toward gene therapy}

In this issue of the JCI, Pasmooij et al. (14) convincingly demonstrate that several distinct revertant mutational events can occur in one individual at different time points in life, all contributing to amelioration of the disease phenotype by different mechanisms (14). This notion is supported by recent observations in other hereditary diseases such as tyrosinemia type I (24) and Omenn syndrome (25).

The two patients presented here had autosomal recessive non-Herlitz junctional EB caused by compound heterozygous or homozygous germline mutations in the laminin $\beta 3$ (LAMB3) gene, respectively (14). Molecular analysis of biopsy specimens derived from areas of healthy-appearing skin led to the identification of five different correcting somatic mutations that predominantly affected mRNA splicing. Interestingly, one of the patients studied by these authors revealed expansion of the healthy skin regions harboring a spontaneously occurring correcting second-site mutation. It is tempting to speculate that some of the keratinocytes in which revertant mutational events occurred must have been epidermal stem cells. Over time, clonal expansion of these stem cells led to amelioration of the phenotype within a defined area of skin due to a selection advantage of revertant stem cells compared with their deficient counterparts (14).
These observations have far-reaching consequences for possible future strategies of gene therapy for autosomal recessive cutaneous disorders. In mosaic individuals, autologous skin grafts derived from areas with normal-appearing skin could be transplanted to affected de-epidermized skin regions on the same patient, thereby following the example of natural gene therapy and avoiding immunologic reactions that might cause graft rejection. Pasmooij et al. (14) conclude that in LAMB3 revertant mosaicism, one might expand in vitro the patient's own revertant cells and use such naturally corrected cells for grafting.

\section{Gene therapy in EB: where are we now?}

When designing gene therapy approaches as a future method for causal treatment of severe inherited cutaneous diseases some 10-15 years ago, investigators convinced of the benefits of this therapeutic regimen were often confronted with skepticism and concerns. Today, gene therapy for EB is no longer wishful thinking but is at the verge of being introduced into clinical practice.

Very recently, Mavilio et al. (26) reported the first successful ex vivo gene therapy approach in a patient suffering from LAM5- $\beta 3$-deficient junctional EB, a disorder that is severe and often lethal due to dysfunctional skin adhesion, manifesting with extensive blistering at birth and serious complications such as recurrent infections. In a phase I/II clinical trial, epidermal stem cells from this patient were transduced with a retroviral vector expressing LAMB3 cDNA (encoding LAM5- $\beta 3$ ) and used to create genetically corrected epidermal grafts. After surgical preparation, these grafts were transplanted onto the patient's legs, resulting in development of fully functional and adherent epidermis. One year later, the grafts were stable and did not show any blistering, indicating that this therapeutic approach was curative (26).

This encouraging report (26) is just the beginning of a new era in which laboratory researchers and clinicians will intensify their efforts to develop and improve strategies of gene therapy for potentially fatal skin diseases. The concept of revertant mosaicism as presented in this issue by Pasmooij et al. (14) will be an important contribution toward this goal. In particular, the discovery that postzygotic mutations giving rise to revertant mosaicism occur far more frequently than so far assumed may serve as a starting point to develop new strategies of gene therapy that we are sorely missing in our clinical practice today.

\section{Acknowledgments}

J. Frank is member of the Network Epidermolysis Bullosa (EB) and the Network for Ichthyoses and Related Keratinization Disorders (NIRK), which are supported by Network grants from the Federal Ministry of Education and Research (BMBF), Germany. R. Happle is an advisor for NIRK.

Address correspondence to: Jorge Frank, Maastricht University Centre for Molecular Dermatology (MUCMD), Department of Dermatology, University Hospital Maastricht, P. Debyelaan 25; Postbus 5800, 6202 AZ Maastricht, The Netherlands. Phone: 31-43-3875292; Fax: 31-43-3877293; E-mail: jfra@sder.azm.nl.

1. McKusick, V.A. 1973. Genetics and dermatology or if I were to rewrite Cockayne's Inherited abnormalities of the skin. J. Invest. Dermatol. 60:343-359.

2. McGrath, J.A. 2004. Translational benefits from research on rare genodermatoses. Australas. J. Dermatol. 45:89-93.

3. Irvine, A.D., and McLean, W.H. 2003. The molecular genetics of the genodermatoses: progress to date and future directions. Br. J. Dermatol. 148:1-13.

4. Happle, R. 1995. What is a nevus? A proposed definition of a common medical term. Dermatology. 191:1-5.

5. Happle, R. 2006. X-chromosome inactivation: role in skin disease expression. Acta Paediatr. Suppl. 95:16-23.

6. Yochem, J., Sundaram, M., and Bucer, E.A. 2000. Mosaic analysis in Caenorhabditis elegans. Methods Mol. Biol. 135:447-462.

7. Blair, S.S. 2003. Genetic mosaic techniques for studying Drosophila development. Development. 130:5065-5072.

8. Rossant, J., and Spence, A. 1998. Chimeras and mosaics in mouse mutant analysis. Trends Genet. 14:358-363.

9. Carmany-Rampey, A., and Moens, C.B. 2006. Modern mosaic analysis in the zebrafish. Methods. 39:228-238.

10. Happle, R. 2002. Dohi Memorial Lecture: new aspects of cutaneous mosaicism. J. Dermatol. 29:681-692.

11. Poblete-Gutiérrez, P., et al. 2004. Allelic loss underlies type 2 segmental Hailey-Hailey disease, providing molecular confirmation of a novel genetic concept. J. Clin. Invest. 114:1467-1474. doi:10.1172/ JCI200421791.

12. Happle, R. 1996. Segmental forms of autosomal dominant skin disorders: different types of severity reflect different states of zygosity. Am. J. Med. Genet. 66:241-242.

13. Happle, R., and König, A. 1999. Dominant traits may give rise to paired patches of either excessive or absent involvement. Am. J. Med. Genet. 84:176-177.

14. Pasmooij, A.M.G., Pas, H.H., Bolling, M.C., and Jonkman, M.F. 2007. Revertant mosaicism in junctional epidermolysis bullosa due to multiple correcting second-site mutations in $L A M B 3$. J. Clin. Invest. 117:1240-1248. doi:10.1172/JCI30465.

15. Hafner, C., et al. 2006. Mosaicism of activating FGFR3 mutations in human skin causes epidermal nevi. J. Clin. Invest. 116:2201-2207. doi:10.1172/JCI28163. 
16. Meyer, M., and Wagner, E. 2006. Recent developments in the application of plasmid DNA-based vectors and small interfering RNA therapeutics for cancer. Hum. Gene Ther. 17:1062-1076.

17. Xia, X., Zhou, H., Huang, Y., and Xu, Z. 2006. Allele-specific RNAi selectively silences mutant SOD1 and achieves significant therapeutic benefit in vivo. Neurobiol. Dis. 23:578-586.

18. Yang, T.P., Stout, J.T., Patel, P.I., Alsford, R.L., and Caskey, C.T. 1988. Spontaneous reversion of novel LeschNyhan mutation by HPRT gene rearrangement. Somat. Cell Mol. Genet. 14:293-303.

19. Jonkman, M.F. 1999. Revertant mosaicism in human genetic disorders. Am. J. Med. Genet, 85:361-364.
20. Bittar, M., and Happle, R. 2005. Revertant mosaicism and retrotransposons: another explanation of "natural gene therapy". Am. J. Med. Genet. A. 137:222.

21. Pizzuti, A., Pieretti, M., Fenwick, R.G., Gibba, R.A., and Caskey, C.T. 1992. A transposon-like element in the deletion-prone region of the dystrophin gene. Genomics. 13:594-600.

22. Thanh, L.T., Nguyen, T.M., Helliwell, T.R., and Morris, G.E. 1995. Characterization of revertant muscle fibers in Duchenne muscular dystrophy, using exon-specific monoclonal antibodies against dystrophin. Am. J. Hum. Genet. 56:725-731.

23. Jonkman, M.F., et al. 1997. Revertant mosaicism in epidermolysis bullosa caused by mitotic gene conversion. Cell. 88:543-551.

24. Bliksrud, Y.T., Brodtkorb, E., Andresen, P.A., van den Berg, I.E.T., and Kvittingen, E.A. 2005. Tyrosinaemia type I - de novo mutation in liver tissue suppressing an inborn splicing defect. J. Mol. Med. 83:406-410.

25. Wada, T., et al. 2005. Oligoclonal expansion of T lymphocytes with multiple second-site mutations leads to Omenn syndrome in a patient with RAG1deficient severe combined immunodeficiency. Blood. 106:2099-2101.

26. Mavilio, F., et al. 2007. Correction of junctional epidermolysis bullosa by transplantation of genetically modified epidermal stem cells. Nat. Med. 12:1397-1402.

\title{
Cellular and molecular basis of wound healing in diabetes
}

\author{
Harold Brem ${ }^{1}$ and Marjana Tomic-Canic ${ }^{2}$ \\ ${ }^{1}$ Wound Healing and Vascular Biology Laboratory, Division of Plastic Surgery, Columbia University College of Physicians and Surgeons, \\ New York, New York, USA. ${ }^{2}$ Tissue Repair Laboratory, Tissue Engineering, Regeneration, and Repair Program, and \\ Department of Dermatology, Hospital for Special Surgery, Weill Medical College of Cornell University, New York, New York, USA.
}

\begin{abstract}
Diabetic foot ulcers (DFUs), a leading cause of amputations, affect $15 \%$ of people with diabetes. A series of multiple mechanisms, including decreased cell and growth factor response, lead to diminished peripheral blood flow and decreased local angiogenesis, all of which can contribute to lack of healing in persons with DFUs. In this issue of the JCI, Gallagher and colleagues demonstrate that in diabetic mice, hyperoxia enhances the mobilization of circulating endothelial progenitor cells (EPCs) from the bone marrow to the peripheral circulation (see the related article beginning on page 1249). Local injection of the chemokine stromal cell-derived factor-1 $\alpha$ then recruits these EPCs to the cutaneous wound site, resulting in accelerated wound healing. Thus, Gallagher et al. have identified novel potential targets for therapeutic intervention in diabetic wound healing.
\end{abstract}

Diabetes affects approximately 170 million people worldwide, including 20.8 million in the USA (1), and by 2030 these numbers are projected to double (2). The foot ulcer is a leading cause of hospital admissions for people with diabetes in the developed world (3) and is a major morbidity associated with diabetes, often leading to pain, suffering, and a poor quality of life for patients. Diabetic foot ulcers (DFUs) are estimated to occur in $15 \%$ of all patients with diabetes (3) and precede $84 \%$ of all diabetes-related lowerleg amputations (4).

Nonstandard abbreviations used: DFU, diabetic foot ulcer; EPC, endothelial progenitor cell; HBO, hyperbaric oxygen therapy; SDF-1 $\alpha$, stromal cell-derived factor $-1 \alpha$.

Conflict of interest: The authors have declared that no conflict of interest exists.

Citation for this article: J. Clin. Invest. 117:1219-1222 (2007). doi:10.1172/JCI32169.
Despite the existence of protocols to standardize care, the physiological impairments that can result in a DFU complicate the healing process. Currently, the only FDAapproved growth factor and cell therapies for DFUs are not routinely used during treatment, preventing professionals from implementing evidence-based protocols (5).

\section{Molecular pathogenesis of diabetic wound healing}

The moment a person with diabetes suffers a break in the skin of their foot, they become at danger for amputation. Most commonly, patients have neuropathy, which could be causative. When coupled with an impaired ability to fight infection, these patients become largely unable to mount an adequate inflammatory response. Thus, the DFU that may look like a healing wound becomes a portal for infection that can lead to sepsis and require limb amputation.
Over 100 known physiologic factors contribute to wound healing deficiencies in individuals with diabetes (Figure 1). These include decreased or impaired growth factor production (6-8), angiogenic response $(8,9)$, macrophage function (10), collagen accumulation, epidermal barrier function, quantity of granulation tissue (8), keratinocyte and fibroblast migration and proliferation, number of epidermal nerves (11), bone healing, and balance between the accumulation of ECM components and their remodeling by MMPs (12). Wound healing occurs as a cellular response to injury and involves activation of keratinocytes, fibroblasts, endothelial cells, macrophages, and platelets. Many growth factors and cytokines released by these cell types are needed to coordinate and maintain healing.

Molecular analyses of biopsies from the epidermis of patients have identified pathogenic markers that correlate with delayed wound healing. These include overexpression of c-myc and nuclear localization of $\beta$-catenin (13). Coupled with a reduction in and abnormal localization of EGFR and activation of the glucocorticoid pathway, keratinocyte migration is inhibited $(13,14)$. At the nonhealing edge (callus) of DFUs, keratinocytes show an absence of migration, hyperproliferation, and incomplete differentiation $(13,14)$. Fibroblasts demonstrate a phenotypic change as well as decreased migration and proliferation. 